\title{
SIRT1 directly activates autophagy in human chondrocytes
}

\author{
Pradeep K. Sacitharan (1) ${ }^{1,2}$, George Bou-Gharios ${ }^{2}$ and James R. Edwards ${ }^{2}$
}

\begin{abstract}
Osteoarthritis $(\mathrm{OA})$ is the most common form of arthritis worldwide with no effective treatment. Ageing is the primary risk factor for OA. We sought to investigate if there is a distinct and functional convergence of ageing-related mechanisms SIRT1 and autophagy in chondrocytes. Our results show that, levels of SIRT1 are decreased in human normal aged and OA cartilage compared with young cartilage. Moreover, silencing SIRT1 in chondrocytes lead to decreased expression of chondrogenic markers but did not alter the expression of catabolic proteases. In contrast, activation of SIRT1 increased autophagy in chondrocytes by the deacetylation of lysine residues on crucial autophagy proteins (Beclin1, ATG5, ATG7, LC3). This activation was shown to be mTOR/ULK1 independent. Our results indicate that maintenance of autophagy in chondrocytes by SIRT1 is essential for preserving cartilage integrity throughout life and therefore is a target for drug intervention to protect against OA.
\end{abstract}

\section{Introduction}

Osteoarthritis $(\mathrm{OA})$ is the most common form of arthritis $^{1}$. There are no effective therapeutic options for $\mathrm{OA}$ and joint replacement still remains the most common approach to treat the disease. The primary risk factor for OA is increased age, where over time cartilage tissue degrades. SIRT1, a class III histone deacetylase, controls lifespan extension and age-related cellular mechanisms including stress responses, circadian rhythm and genetic repair ${ }^{2}$. Decreased expression of SIRT1 is linked with ageassociated disease (e.g., neurodegeneration, ischaemic heart disease, diabetes) ${ }^{3}$ while pharmacological stimulation increases lifespan and prevents age-related disorders $^{4}$. Observational studies have also linked SIRT1 to abnormal chondrocyte biology and $\mathrm{OA}^{5-7}$. Similarly, the process of autophagy (self-eating), where unwanted proteins are degraded and recycled, decreases with age and $\mathrm{OA}$ and is linked to the pathobiology of ageing-related disorders $^{8-10}$.

\footnotetext{
Correspondence: James R. Edwards (James.Edwards@ndorms.ox.ac.uk) ${ }^{1}$ Botnar Research Centre, University of Oxford, Oxford OX3 7LD, UK

${ }^{2}$ The Institute of Ageing and Chronic Disease, University of Liverpool, Liverpool L7 8TX, UK

Edited by I. Amelio
}

Recent evidence draws associations between both ageing-related processes, where SIRT1 significantly increased autophagy in aged chondrocytes, while reduced expression of SIRT1 lowered autophagy in young chondrocytes ${ }^{11}$. SIRT1 activation of autophagy may be linked to microRNAs or the AMPK/mTOR pathways in chondrocytes $^{12,13}$. However, the direct regulation of autophagy in cartilage by SIRT1 are still unclear ${ }^{11-13}$. The aim of this study was to investigate if SIRT1 directly binds and deacetylates autophagy proteins in chondrocytes and if SIRT1 positively influences chondrogenic markers. The dysregulation of such chondrogenic markers are common in ageing cartilage cells and OA tissues.

Here, we show SIRT1 levels decrease in human OA which in turn decreased chondrogenic markers alongside key autophagy markers. Activation of SIRT1 increased chondrocyte autography alongside chondrogenesis which suggests a beneficial role of the SIRT1-autophagy axis in chondrocytes. Most importantly, we show SIRT1 to bind and directly deacetylase several autophagy proteins (ULK1, BECLIN1, ATG5, ATG7 and LC3 I/II). These results suggest a direct action of SIRT1 upon autophagylinked proteins in chondrocytes, the targeting of which

\section{(c) The Author(s) 2020}

(c) Open Access This article is licensed under a Creative Commons Attribution 4.0 International License, which permits use, sharing, adaptation, distribution and reproduction in any medium or format, as long as you give appropriate credit to the original author(s) and the source, provide a link to the Creative Commons license, and indicate if changes were made. The images or other third party material in this article are included in the article's Creative Commons license, unless indicated otherwise in a credit line to the material. If material is not included in the article's Creative Commons license and your intended use is not permitted by statutory regulation or exceeds the permitted use, you will need to obtain permission directly from the copyright holder. To view a copy of this license, visit http://creativecommons.org/licenses/by/4.0/. 
may be beneficial for enhanced chondrogenesis and the prevention of OA.

\section{Results}

Loss of SIRT1 expression disrupts cartilage homoeostatic markers

Normal human cartilage samples from aged individuals showed decreased SIRT1 protein levels compared with young and were further decreased in OA samples (Fig. 1a, b).
SIRT1 siRNA knockdown in healthy isolated human articular chondrocytes (HACs) (Fig. 1c-g) and HTB-94 cells (Supplementary Fig. S1a-e), reduced chondrogenic markers, SOX9, type 2 collagen (COL2A1) and aggrecan $(A C A N)$. In support of these findings, pharmacological inhibition (EX-527) of SIRT1 reduced the expression of COL2A1, ACAN and SOX-9 (Fig. 1h-1). Conversely, the expression of the key chondrogenic markers increased when SIRT1 activity was stimulated by SRT1720 (Fig. 1h-l).
A

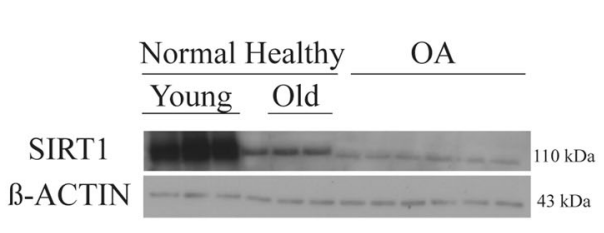

D

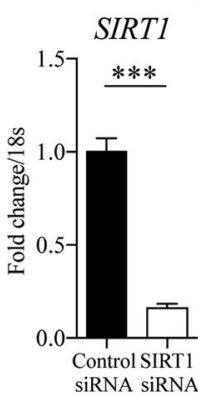

E

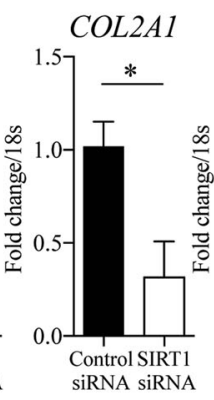

F

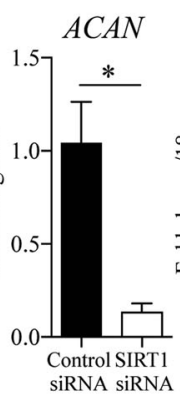

B

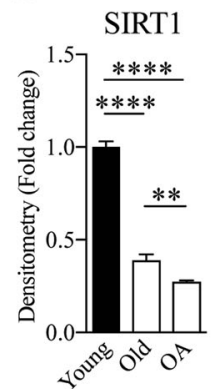

C

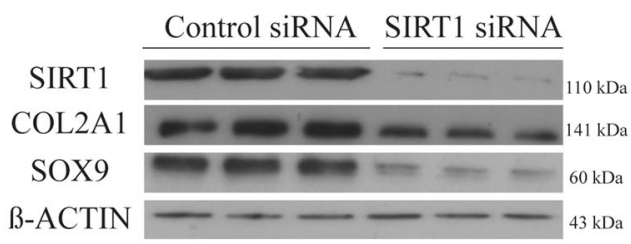

H
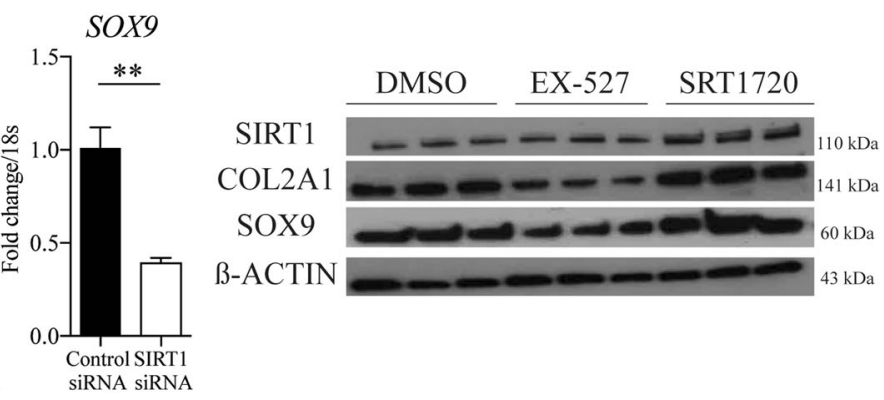

I

J

K

L

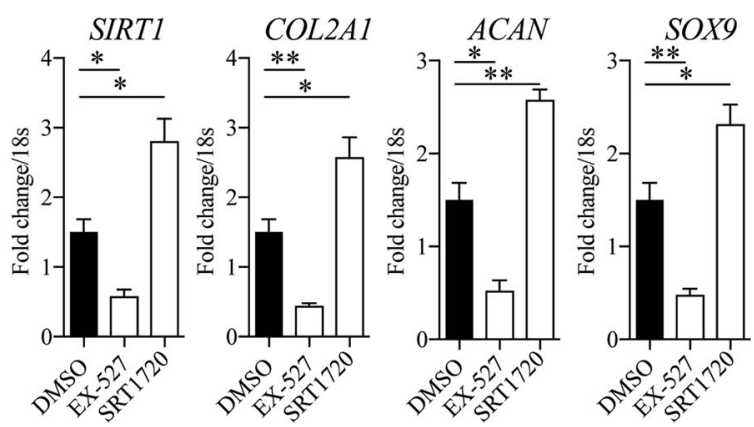

Fig. 1 Loss of SIRT1 expression disrupts cartilage homoeostatic markers. a SIRT1 protein expression (b) and quantification in isolated chondrocytes from young healthy (21-37 years), old (62-68 years) and OA (49-86 years) knee joints. c Protein expression of cartilage markers in isolated chondrocytes from young healthy (21-37 years) transfected with SIRT1 siRNA or control siRNA ( $n=3$ ). $\mathbf{d}-\mathbf{g}$ RT-qPCR analysis of cartilage marker expressions in isolated chondrocytes from young healthy (21-37 years) following SIRT1 siRNA or control siRNA transfection ( $n=3)$. $\mathbf{h}$ Protein expression of cartilage marker expressions in HTB-94 cells with DMSO control or EX-527 (100 nM) or SRT1720 (500 nM) ( $n=3$ ). i-I RT-qPCR analysis of cartilage marker expressions in HTB-94 cells treated with DMSO control or EX-527 (100 nM) or SRT1720 (500 nM) ( $n=3$ ). All RT-qPCR gene expressions were normalised to the endogenous level of $18 \mathrm{~s}$. All data are expressed as mean \pm S.E.M of $n$ observations. Students unpaired $t$-test or ANOVA with Tukeys comparison were used for statistical analysis. NS $=$ non-significant. $p<0.05, p<0.01, p<0.001$ or $p<0.0001$ represented in all figures as *,**, *** or **** respectively. 


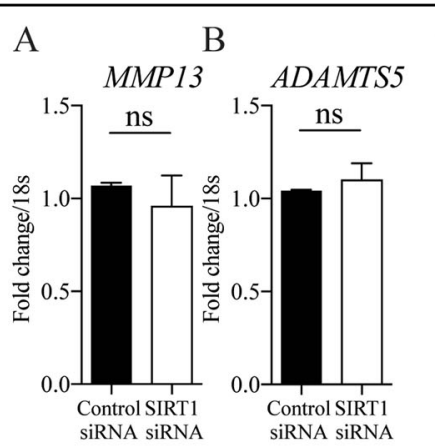

C

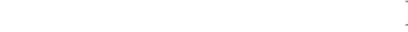

SIRT1 SiRNA

IL-1B

SIRT1

MMP13

ADAMTS5

B-ACTIN

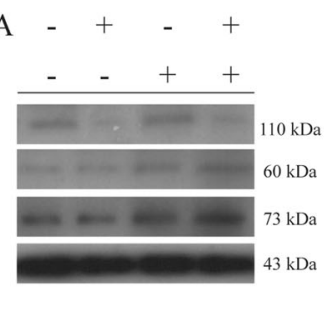

G
D

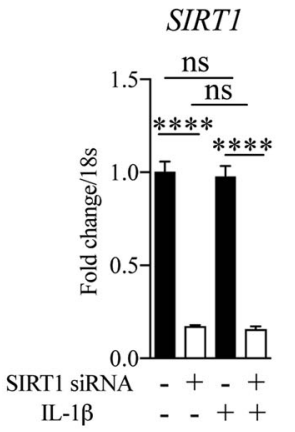

$\mathrm{H}$
E

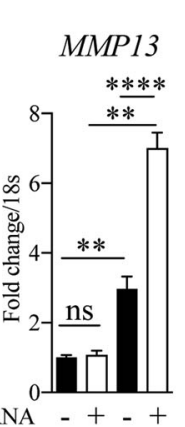

F

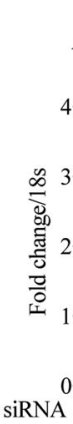

ADAMTS5

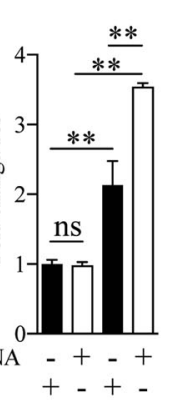
IL-1 $\beta+$ - +
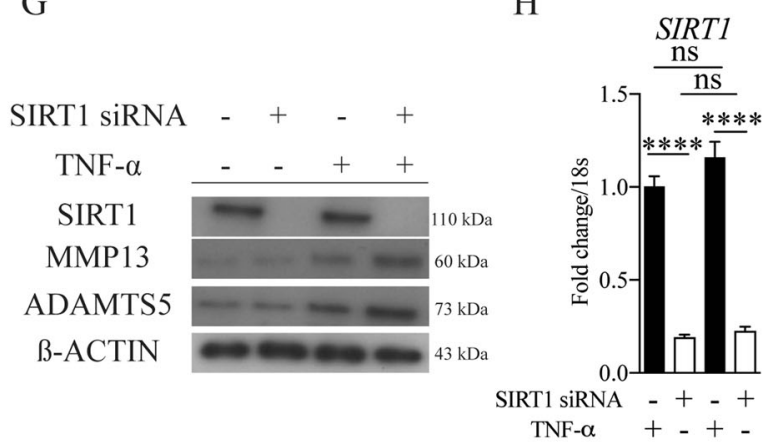

I

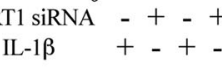

$\begin{array}{cc}\text { SIRT1 SiRNA } & -+ \\ \text { TNF- } \alpha & +\end{array}$

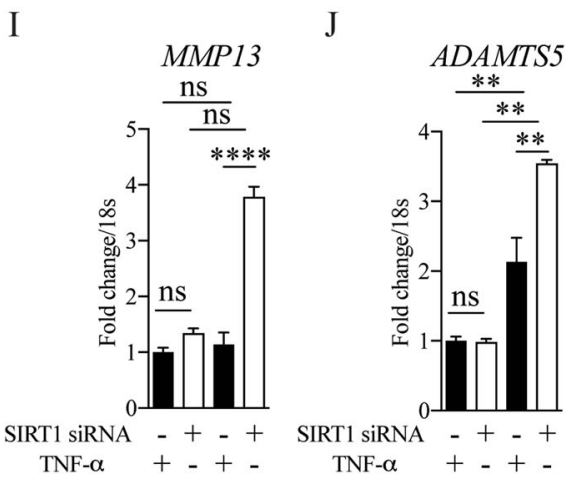

Fig. 2 Silencing of SIRT1 does not affect major catabolic enzymes in chondrocytes. a, b mRNA expression of catabolic proteases in HTB-94 cells following SIRT1 siRNA or control siRNA transfection $(n=3)$. $\mathbf{c}$ protein expression and $(\mathbf{d}-\mathbf{f})$ mRNA of SIRT1, MMP13 and ADAMTS-5 in isolated chondrocytes from young healthy (21-37 years) treated with SIRT1 siRNA or control siRNA+/-IL1- $\beta(n=3)$. $\mathbf{g}$ protein expression $(\mathbf{h}-\mathbf{j})$ and mRNA of SIRT1, MMP13 and ADAMTS-5 in isolated chondrocytes from young healthy (21-37 years) treated with SIRT1 siRNA or control siRNA $+/-$ TNF- $(n=3)$. All RT-qPCR gene expressions were normalised to the endogenous level of $18 \mathrm{~s}$. All data are expressed as mean \pm S.E.M of $n$ observations. Students unpaired $t$-test or ANOVA with Tukeys comparison were used for statistical analysis. NS $=$ non-significant. $p<0.01$ or $p<0.0001$ represented in all figures as ${ }^{* *}$ or ${ }^{* * *}$ respectively.

\section{Silencing of SIRT1 does not affect major catabolic enzymes in chondrocytes}

The maintenance of cartilage integrity and OA-disease progression are both associated with an adequate balance of chondrocyte formation and function, and local levels of matrix-degrading proteases. Silencing SIRT1 did not affect the expression of the critical extracellular matrix (ECM) degrading proteases $M M P-13$ and ADAMTS-5 in HACs (Fig. 2a, b). However, loss of SIRT1 augmented the increase in $M M P-13$ and
ADAMTS-5 gene and protein expression induced by catabolic stimuli (IL-1 $\beta$ or TNF $\alpha$ ) above that seen in control cells (Supplementary Fig. S2c-j).

\section{Chondrocyte autophagy is SIRT1 dependent}

We next examined the role of autophagy in aged and diseased cartilage, and its putative link with SIRT1. Interestingly, the gene and protein expression of critical autophagy-related genes ULK1, BECLIN1 and LC3, were regulated in line with molecular (Fig. $3 a-e$ and 


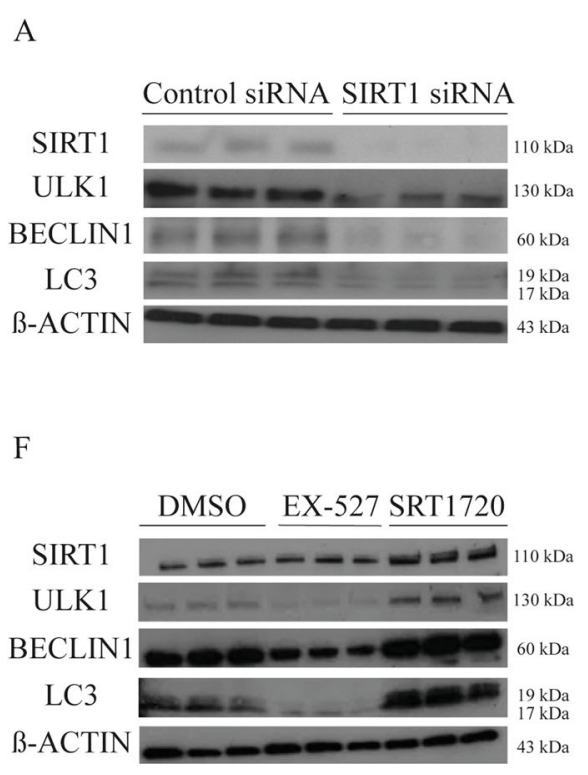

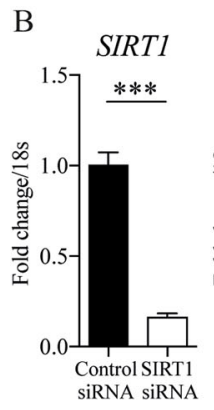

G

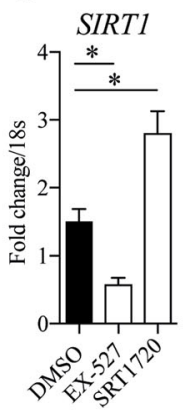

L

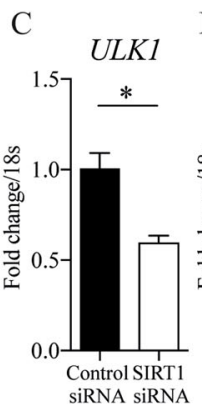

$\mathrm{H}$

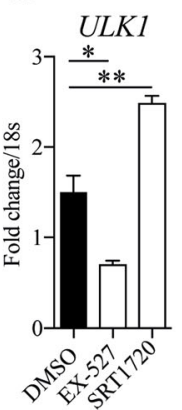

M

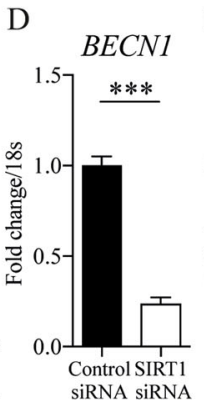

I

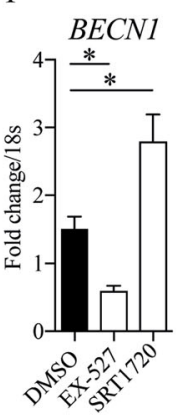

E $\quad L C 3$

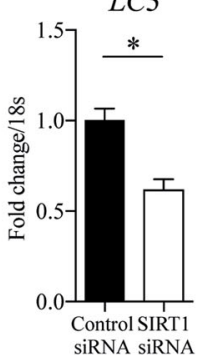

$\mathrm{J}$

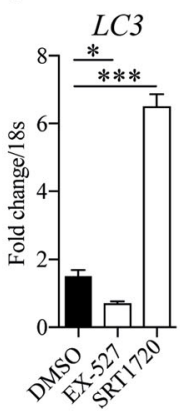

K

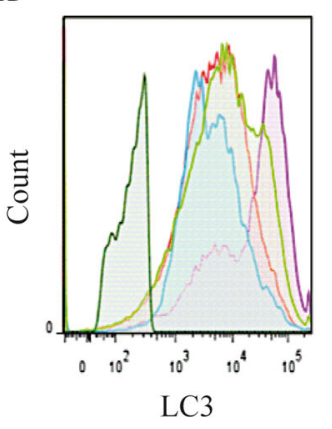

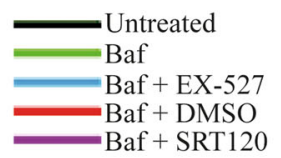

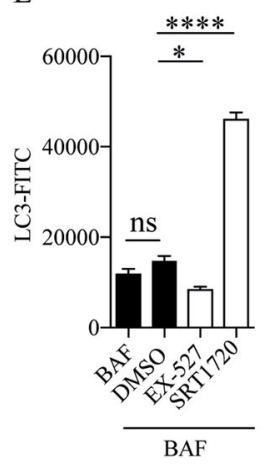

$\mathrm{N}$

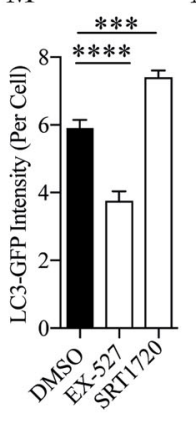

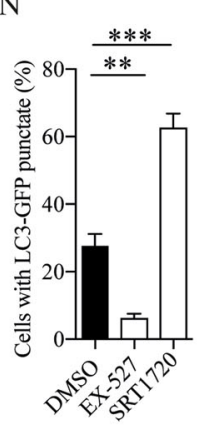

Fig. 3 Chondrocyte autophagy is dependent on SIRT1. a Protein levels and (b-e) RT-qPCR analysis of autophagy marker expression in isolated chondrocytes from young healthy (21-37 years) transfected with SIRT1 siRNA or control siRNA $(n=3)$. f Protein expression of cartilage marker expressions in HTB-94 cells with DMSO control or EX-527 (100 nM) or SRT1720 (500 nM) $(n=3)$. g-j RT-qPCR analysis of cartilage marker expressions in HTB-94 cells treated with DMSO control or EX-527 (100 nM) or SRT1720 (500 nM) ( $n=3$ ). k Histogram and (I) quantification of mean fluorescence intensity (MFI) of LC3-II in HTB-94 cells either untreated or treated with bafilomycin A1 (BAF; $10 \mathrm{nM}$ ), alongside DMSO (vehicle control), for $2 \mathrm{~h}$ ( $n=3$ ). m Quantification of total LC3-GFP intensity per chondrocyte and (n) percentage of chondrocytes with LC3 positive punctate from cartilaginous femoral heads of LC3-GFP mice. Femoral heads were treated with either DMSO control or EX-527 (100 nM) or SRT1720 (500 nM) for 2 h before fixation ( $n=30-50$ cells from 3 mice per group). All RT-qPCR gene expressions were normalised to the endogenous level of $18 \mathrm{~s}$. All data are expressed as mean \pm S.E.M of $n$ observations. Students unpaired $t$-test or ANOVA with Tukeys comparison were used for statistical analysis. NS $=$ non-significant. $p<0.05, p<0.01, p<0.001$ or $p<0.0001$ represented in all figures as ***,*** or **** respectively.

supplementary Fig. S2a-e) and pharmacological alteration of SIRT1 (Fig. 3f-j). In support of this data, SIRT1induced changes in autophagy were quantified in treated chondrocytes using flow cytometry and by assessing LC3GFP intensity. The conversion from LC3-I to LC3-II is indicative of increased autophagy ${ }^{14}$. LC3 II mean fluorescent intensity (MFI) increased by $213.5 \%$ when SIRT1 was activated, whereas inhibiting SIRT1 decreased significantly LC3 II MFI by $42.17 \%$ (Fig. 3k, 1). This finding was supported by studies using LC3-GFP reporter mice, where isolated primary chondrocytes treated as above, showed decreased LC3-GFP + puncta (54.37\%) and staining intensity per cell (66.8\%) following SIRT1 inhibition, and a $20.5 \%$ increase in LC3-GFP + puncta and staining per cell (28.0\%) upon stimulation (Fig. 3m, $\mathrm{n}$ and supplementary Fig. S3f, g). 
A

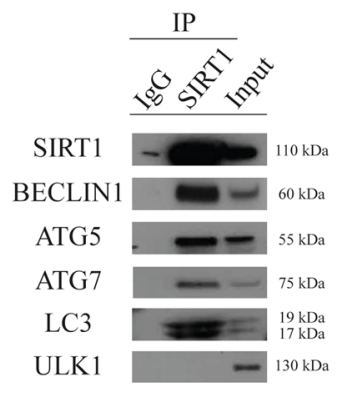

C

IP

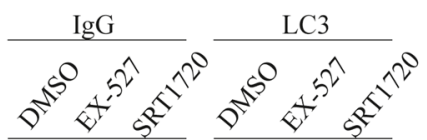

ACETYL-LYS

LC3

B-ACTIN (IN)

E

IP
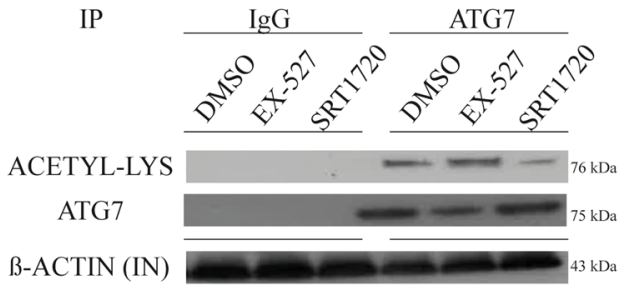

I

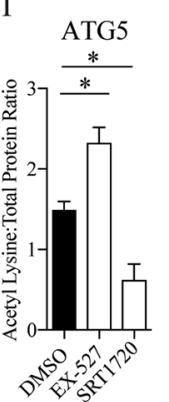

B
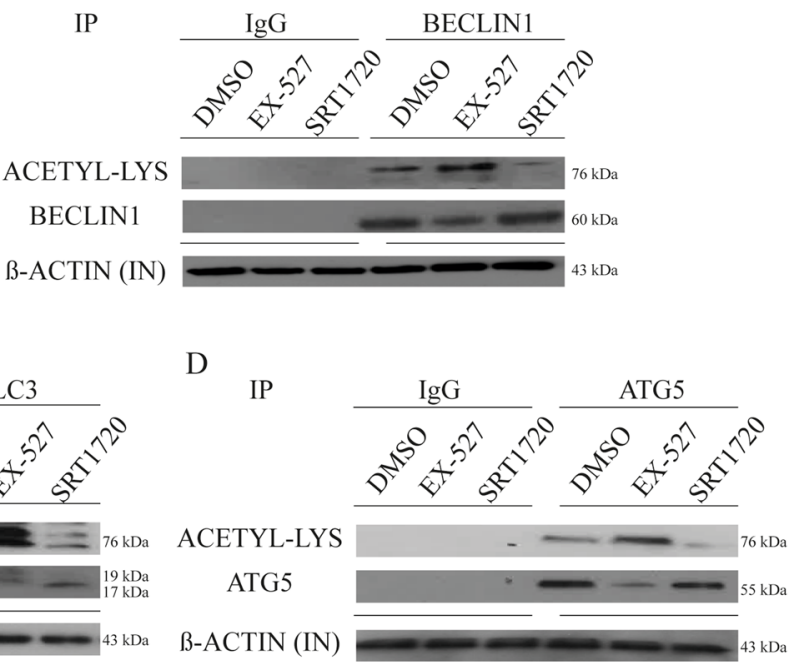

F

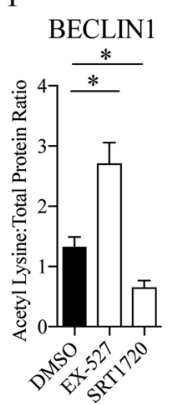

G

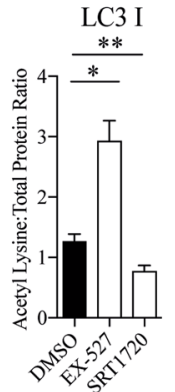

$\mathrm{H}$

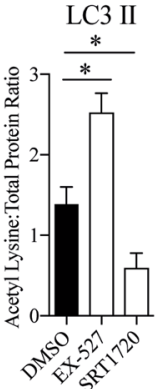

Fig. 4 SIRT1 directly interacts and activates autophagy proteins in chondrocytes. a Western blot showing the binding of autophagy markers using immunoprecipitation assays against SIRT1 in HTB-94 cells $(n=3)$. b-e Western blots showing representative acetylation status (total acetyl lysine) and total protein expression of immunoprecipitated (IP) Atg5, Atg7, BECLIN1 and LC3 in HTB-94 cells treated with DMSO (control), EX-527 (100 nM) or SRT1720 (500 nM). $\beta$-Actin from input (IN) lysate is shown as loading control $(n=3)$. $\mathbf{f}-\mathbf{j}$ Ratio of acetyl lysine compared to total protein of autophagy markers in HTB-94 cells treated with DMSO (control), EX-527 (100 nM) or SRT1720 (500 nM) ( $n=3$ ). k SIRT1 and autophagy are decreased in aged and OA tissue. Activating SIRT1 in chondrocytes increases autophagy by direct deacetylation. All data are expressed as mean \pm S.E.M of $n$ observations. ANOVA with Tukeys comparison was used. $p<0.05$ or $p<0.01$ represented in figures as * or ** respectively.

\section{SIRT1 directly interacts and activates autophagy proteins in chondrocytes}

To ascertain whether changes in SIRT1 and autophagy were linked or occurring independently, immunoprecipitation analysis was performed to determine which autophagy-related proteins directly interact with SIRT1. Several autophagy mediators including BECLIN1, ATG5, ATG7 and LC3, but excluding ULK1, were shown to bind the SIRT1 protein (Fig. 4a). Moreover, each protein was functionally modified through its interaction with SIRT1 
demonstrated by increased acetylation of common representative sites when SIRT1 was pharmacologically blocked (EX-527), and by decreased acetylation in the presence of a SIRT1 activator (SRT1720) (Fig. 4b-k and supplementary Fig. S3a).

\section{Discussion}

We have shown that both SIRT1 and autophagy are similarly dysregulated in human chondrocytes from ageing and OA cartilage that a direct functional relationship exists between both longevity-linked factors. Collectively, this data suggests that the decreasing levels of SIRT1 in human chondrocytes with increasing age, and further loss of expression in OA samples may underlie the pathogenesis of $\mathrm{OA}$ and decreased cartilage integrity during ageing. The convergence of two accepted ageing-related mechanisms in the pathogenesis of osteoarthritis therefore seems highly likely.

The sirtuin family of deacetylase enzymes are known to be dependent on the local availability of Nicotinamide adenine dinucleotide $(\mathrm{NAD}+)$ for efficient activity to occur. Consequently, metabolic alterations resulting in leading to changes in the NADH/NAD + ratio, have potential to indirectly impact the range of cellular processes controlled by Sirtuin proteins, such as mitochondrial biogenesis and insulin sensitivity, and which includes SirT1 activity. Interestingly, the loss of SIRT1 and the $\mathrm{NAD}^{+}$co-factor, are shown to be decreased in OA patients and experimental models of bone and joint disease $^{5-7,15-17}$. Recent observational studies in mice suggest that loss of SIRT1 in all chondrocytes through use of the type II collagen promoter, predisposed to OA development at 1 year $^{18}$. Interestingly, our studies show impaired COL2A $1^{19}$, SOX- $9^{20}$ and ACAN ${ }^{21}$ expression but no early change in MMP-13 or ADAMTS5 expression following SIRT1 deletion in HACs. As shown by other studies MMP-13 and ADAMTS-5 only changed following a catabolic stimuli (IL-1 $\beta$ or TNF $\alpha$ ) alongside SIRT1 loss ${ }^{5,22}$. This suggests the regulation of NFKB activation by SIRT1 is an important mechanism in response to catabolic stimuli in cells including chondrocytes ${ }^{23,24}$. Our results also suggest increased protease activity occurs following a reduction in SIRT1 levels which has in turn, compromised the intrinsic capacity of chondrocytes to function adequately by impairing autophagy. The posttranslational modification of autophagy proteins has recently been reported to exert significant control over autophagic activity ${ }^{25}$. Specifically, elevated acetylation of BECLIN1 reduced autophagosome maturation in cancer cells $^{26}$, increased deacetylation of LC3 promoted autophagy following caloric restriction ${ }^{27}$ and SIRT1 deacetylation of LC3 has been shown to effectively redistribute LC3 in an activated form from nucleus to cytoplasm controlling total LC3 levels ${ }^{28,29}$. This is in accordance with our findings where increased acetylation of key autophagy proteins was brought about by loss of SIRT1. Decreased mTOR/ULK1 signalling also increases autophagy to protect against $\mathrm{OA}^{30,31}$. Here we demonstrate a new role for SIRT1 in targeting downstream autophagic proteins, but interestingly, through the binding and activation of ULK1, which alludes to a separate regulation of autophagy independent of the mTOR/ULK1 signalling pathway.

We also observed SIRT1-mediated changes in mRNA of autophagy markers suggesting SIRT1 might exert transcriptional control of autophagy alongside posttranslational modifications. This might be explained by the direct deacetylation of the autophagy-related Transcription factor EB (TFEB) by SIRT $1^{32}$. SIRT1 deacetylation promotes TFEB activity to increase autophagy ${ }^{33}$. Similarly, SIRT1 has multiple targets ${ }^{34}$ in many cell types including chondrocytes, such as Set7/9, Sox-9, TNF $\alpha^{19,20,35-37}$ which might also influence how SIRT1 pathways connect to or control chondrocyte autophagy in this system and ageing overall.

These data indicate that the convergence of two wellaccepted ageing-related mechanisms may underlie the development of OA. Whilst further rescue studies targeting SIRT1 and autophagy-related proteins in OA tissues will provide valuable information indicating a causative role in OA pathogenesis, this work has identified new direct interactions between SIRT1 and autophagyrelated proteins where functional modifications and changes in autophagic flux are dependent upon SIRT1 expression in chondrocytes. Moreover, the data suggests that declining expression of SIRT1 with increasing age predisposes to OA by impairing autophagy. Targeting the SIRT1-autophagy axis pharmacologically may therefore preserve cartilage integrity, and represent a viable, costeffective and well tolerated approach to the management of OA.

\section{Materials and methods \\ Isolation of human chondrocytes}

Healthy or OA knee/hip cartilage was obtained from surgical patients (21-86 years old). Tissue samples were collected with informed donor consent in full compliance with national and institutional ethical requirements, the United Kingdom Human Tissue Act, and the Declaration of Helsinki. Dissected cartilage pieces were incubated overnight in Dulbecco's modified Eagle's medium (DMEM) with $1 \mathrm{mg} / \mathrm{ml}$ Collagenase A (Roche Pharmaceuticals) at $37^{\circ} \mathrm{C}$ for $5-6 \mathrm{~h}$ to isolate cells. Cells used in experiments were at passage 1 .

\section{Cell culture}

HTB-94 chondrosarcoma cells were obtained from the American Type Culture Collection cultured in DMEM containing $4.5 \mathrm{~g} / \mathrm{l}$ of glucose and L-glutamine (Lonza), 
10\% foetal calf serum (PAA Laboratories), 1\% Penicillin and Streptomycin (Cambrex), Amphotericin B (Gibco) and HEPES solution (Cambrex). Cells were negative for mycoplasma contamination.

\section{SIRT1 siRNA transfection}

Healthy isolated human chondrocytes or HTB-94 cells were wet reverse transfected with Dharmacon ONTARGETplus SMARTpool (4 oligos) human SIRT1 siRNA (25 nM) (Dharmacon Technologies). A scramble oligo sequence was used as control $(25 \mathrm{nM})$ (Dharmacon Technologies).

\section{Drug and cytokine treatments}

Cells were seeded at 300,000 cells per well in 6 well plates in complete DMEM media and cultured containing EX-527 (inhibitor of SIRT1; $100 \mathrm{nM;} \mathrm{Sigma-Aldrich),}$ SRT1720 (activator of SIRT1; $500 \mathrm{nM}$; Selleckchem) or the control of DMSO (Sigma-Aldrich). Forty-eight hours after SIRT1 siRNA transfection, healthy human chondrocytes were treated with $10 \mathrm{ng} / \mathrm{ml}$ of IL-1 $\beta$ (PeproTech) or TNF- $\alpha$ (PeproTech) for $24 \mathrm{~h}$.

\section{FACS}

Autophagy was measured by quantifying LC3-II mean fluorescence intensity using the FlowCellect Autophagy LC3 Antibody-based Assay Kit (Merk-Millipore) according to the manufacturer's instructions after HTB-94 cells were treated with drugs as described above.

\section{Protein analysis}

Cells were homogenised in lysis buffer (RIPA buffer; Sigma-Aldrich), Ethylenediaminetetraacetic acid free protease inhibitor (Roche Pharmaceuticals), Phosphatase inhibitor cocktail 2 and 3 (Sigma-Aldrich) and protein levels quantified by bicinchoninic acid assay (Thermo Fisher Scientific). For western blot analysis samples were probed overnight with primary antibody: Human SIRT1 (\#9475; 1:1000; Cell Signalling Technology, MA, U.S.A), Mouse SIRT1 (07-131; 1:1000; Millipore, Massachusetts, U.S.A) or Beta-actin ( $\beta$-actin) (A2228; 1:20000; SigmaAldrich), COL2A1 (SAB4500366; 1:1000; Sigma-Aldrich), SOX-9 (ab26414; 1:1000; Abcam, Cambridge, U.K.), ULK1 (NBP2-24738; 1:1000; Novus Biologicals, Cambridge, UK), Beclin1 (NB500-249; 1:1000; Novus Biologicals), LC3 (NB100-2220; 1:1000; Novus Biologicals), ATG5 (\#12994; 1:1000; Cell Signalling Technology); ATG7 (\#8558; 1:1000; Cell Signalling Technology), Acetyl-Lysine Antibody (\#9441; 1:1000; Cell Signalling Technology), P62/SQSTM1 (NBP1-48320; 1:1000; Novus Biologicals). Secondary horseradish peroxidase (HRP)conjugated anti-rabbit (\#7074; 1:5000; Cell Signalling Technology), Secondary HRP-conjugated anti-mouse (\#7076; 1:5000; Cell Signalling Technology)

\section{RNA isolation and quantitative PCR}

Total RNA was isolated from cell cultures using RNeasy Mini Kit (Qiagen) as per manufacturer's instructions. RTqPCR was carried out on a $\mathrm{ViiA}^{\mathrm{TM}} 7$ Real-Time PCR System (Applied Biosystems) with TaqMan probes (Supplementary Table 1). Relative gene expression was analysed by the ${ }^{{ }^{\Delta}} \mathrm{Ct}$ method using $18 \mathrm{~s}$ as an endogenous control gene.

\section{LC3-GFP studies}

Femoral heads from LC3-GFP mice (donated by Professor K. Simon, University of Oxford) were avulsed and placed in DMEM media containing either EX-527 $(100 \mathrm{nM})$ or SRT1720 $(500 \mathrm{nM})$ or DMSO. For positive controls, serum free DMEM containing either EX-527 $(100 \mathrm{nM})$ or SRT1720 (500 nM) or DMSO was used. After an incubation of $2 \mathrm{~h}$, femoral heads were embedded in Optimal Cutting Temperature liquid (CellPath) and snapfrozen. Thereafter, samples were cryosectioned at $10 \mu \mathrm{m}$. Fluorescence images were taken using an excitation wavelength of $473 \mathrm{~nm}$ and a band-path of $490-540 \mathrm{~nm}$. Bright field images were obtained using scattering wavelength of $635 \mathrm{~nm}$. The cells were segmented in bright field and GFP intensity was measured in fluorescent channel. Intensity of background was subtracted from intensities of individual cells and divided by intensity of background in order to normalise and score cells. The number of LC3 puncta per cell was counted throughout each $\mathrm{z}$ stacks by two blinded operators.

\section{Immunoprecipitation}

SIRT1 or autophagy-related proteins were immunoprecipitated after $24 \mathrm{~h}$ treatment of HTB-94 cells treated with DMSO (control), EX-527 (100 nM) or SRT1720 (500 nM) using a CO-IP commercially available kit (Pierce). Total acetyl lysine or autophagy targets for the respective experiments were detected by western blotting. $\beta$-Actin from input whole lysate was used as loading control.

\section{Statistical analysis}

All data are expressed as mean \pm standard error of mean (S.E.M) of $\mathrm{n}$ observations. Experiments were statistically analysed utilising the Students unpaired $t$-test for parametric data with independent groups compared with their specific controls or time-matched controls. One-way analysis of variance (ANOVA) with Tukeys comparison test was used to compare 3 or more groups. A significant difference was accepted when $p<0.05, p<0.01, p<0.001$ or $p<0.0001$ represented in all tables and figures as *,**, *** or $* *$ respectively. Data analysis was performed using GraphPad Prism ${ }^{\circledR} 5.0$ (GraphPad Software, California, U.S.A). We calculated sample size according to established power calculations on previous work in the lab, published literature and availability of sample population. 


\section{Acknowledgements}

This work was supported by the Arthritis Research U.K. career developmental fellowship (20631) and Orthopaedic Research U.K. grant (509). Dr Pradeep Kumar Sacitharan was supported by a European Institute of Innovation and Technology transitional fellowship, a U.K.-U.S. all disciplines Fulbright research scholar award, a Daniel Turnberg travel fellowship and a European Molecular Biology Organization (EMBO) short-term fellowship (7941).

\section{Conflict of interest}

The authors declare that they have no conflict of interest.

\section{Publisher's note}

Springer Nature remains neutral with regard to jurisdictional claims in published maps and institutional affiliations.

The online version of this article (https://doi.org/10.1038/s41420-020-0277-0) contains supplementary material, which is available to authorised users.

Received: 25 February 2020 Revised: 4 May 2020 Accepted: 12 May 2020 Published online: 29 May 2020

\section{References}

1. Glyn-Jones, S. et al. Osteoarthritis. Lancet 386, 376-387 (2015).

2. Sacitharan, P. K., Snelling, S. J. B. \& Edwards, J. R. Aging mechanisms in arthritic disease. Discov. Med. 14, 345-352 (2012).

3. Hubbard, B. P. \& Sinclair, D. A. Small molecule SIRT1 activators for the treatment of aging and age-related diseases. Trends Pharmacol. Sci. 35, 146-154 (2014).

4. Baur, J. A., Ungvari, Z., Minor, R. K., Le Couteur, D. G. \& de Cabo, R. Are sirtuins viable targets for improving healthspan and lifespan? Nat. Rev. Drug Discov. 11, 443-461 (2012).

5. Matsushita, T. et al. The overexpression of SIRT1 inhibited osteoarthritic gene expression changes induced by interleukin-1 $\beta$ in human chondrocytes. J. Orthop. Res. 31, 531-537 (2013).

6. Gagarina, V. et al. SirT1 enhances survival of human osteoarthritic chondrocytes by repressing protein tyrosine phosphatase $1 \mathrm{~B}$ and activating the insulin-like growth factor receptor pathway. Arthritis Rheum. 62, 1383-1392 (2010).

7. Gabay, O. et al. Increased apoptotic chondrocytes in articular cartilage from adult heterozygous SirT1 mice. Ann. Rheum. Dis. 1, 613-616 (2012).

8. Caramés, B., Taniguchi, N., Otsuki, S., Blanco, F. J. \& Lotz, M. Autophagy is a protective mechanism in normal cartilage, and its aging-related loss is linked with cell death and osteoarthritis. Arthritis Rheum. 62, 791-801 (2010).

9. Caramés, B., Olmer, M., Kiosses, W. B. \& Lotz, M. K. The relationship of autophagy defects to cartilage damage during joint aging in a mouse model. Arthritis Rheumatol. 67, 1568-1576 (2015).

10. Rubinsztein, D. C., Mariño, G. \& Kroemer, G. Autophagy and aging. Cell 146 682-695 (2011)

11. Liao, F.-X. et al. The new role of Sirtuin1 in human osteoarthritis chondrocytes by regulating autophagy. Cartilage 1947603519847736. https://doi.org/ $10.1177 / 1947603519847736$ (2019).

12. Sun, W., Li, Y. \& Wei, S. miR-4262 regulates chondrocyte viability, apoptosis, autophagy by targeting SIRT1 and activating PI3K/AKT/mTOR signaling pathway in rats with osteoarthritis. Exp. Ther. Med. https://doi.org/10.3892/ etm.2017.5444 (2018).

13. Cetrullo, S. et al. Beneficial effect of 3-hydroxytyrosol on chondrocytes exposed to oxidative stress. Osteoarthr. Cartil. 22, S323-S324 (2014).

14. Tanida, I., Ueno, T. \& Kominami, E. LC3 and autophagy. Methods Mol. Biol. 445 77-88 (2008)
15. Chen, L.-Y. \& Liu-Bryan, R. Effect of nicotinamide riboside on cartilage matrix homeostasis. Osteoarthr. Cartil. 27, S148 (2019).

16. Oh, $\mathrm{H}$. et al. Reciprocal regulation by hypoxia-inducible factor-2a and the NAMPT-NAD ${ }^{+}$-SIRT axis in articular chondrocytes is involved in osteoarthritis. Osteoarthr. Cartil. 23, 2288-2296 (2015).

17. Kobayashi, $\mathrm{H}$. et al. The Nicotinamide Adenine Dinucleotide (NAD)-dependent deacetylase Sirtuin-1 regulates chondrocyte energy metabolism through the modulation of adenosine monophosphate-activated protein kinase (AMPK) in osteoarthritis (OA). J. Arthritis 06, 14-17 (2017).

18. Matsuzaki, T. et al. Disruption of Sirt1 in chondrocytes causes accelerated progression of osteoarthritis under mechanical stress and during ageing in mice. Ann. Rheum. Dis. https://doi.org/10.1136/annrheumdis-2012-202620 (2013).

19. Oppenheimer, $\mathrm{H}$. et al. Set7/9 impacts COL2A1 expression through binding and repression of SirT1 histone deacetylation. J. Bone Miner. Res. 29, 348-360 (2014).

20. Bar, Oz, M. et al. Acetylation reduces SOX9 nuclear entry and ACAN gene transactivation in human chondrocytes. Aging Cell 15, 499-508 (2016).

21. Fujita, $\mathrm{N}$. et al. Potential involvement of SIRT1 in the pathogenesis of osteoarthritis through the modulation of chondrocyte gene expressions. J. Orthop. Res. 29, 511-515 (2011).

22. Moon, M.-H. et al. SIRT1, a class III histone deacetylase, regulates TNF-ainduced inflammation in human chondrocytes. Osteoarthr. Cartil. 21, 470-480 (2013).

23. Kauppinen, A., Suuronen, T., Ojala, J., Kaarniranta, K. \& Salminen, A. Antagonistic crosstalk between NF-KB and SIRT1 in the regulation of inflammation and metabolic disorders. Cell. Signal. 25, 1939-1948 (2013).

24. Edwards, J. R. et al. Silent information regulator (Sir)T1 inhibits NF-KB signaling to maintain normal skeletal remodeling. J. Bone Miner. Res. 28, 960-969 (2013).

25. Popelka, H. \& Klionsky, D. J. Post-translationally-modified structures in the autophagy machinery: an integrative perspective. FEBS J. 282, 3474-3488 (2015).

26. Sun, T. et al. Acetylation of Beclin 1 inhibits autophagosome maturation and promotes tumour growth. Nat. Commun. 6, 7215 (2015)

27. Huang, J. \& Brumell, J. H. Bacteria-autophagy interplay: a battle for survival. Nat Rev. Microbiol. 12, 101-114 (2014).

28. Huang, R. et al. Deacetylation of nuclear LC3 drives autophagy initiation under starvation. Mol. Cell 57, 456-467 (2015).

29. Sacitharan, P. K., Lwin, S., Gharios, G. B. \& Edwards, J. R. Spermidine restores dysregulated autophagy and polyamine synthesis in aged and osteoarthritic chondrocytes via EP300. Exp. Mol. Med. 50, 123 (2018).

30. Zhang, Y. et al. Cartilage-specific deletion of mTOR upregulates autophagy and protects mice from osteoarthritis. Ann. Rheum. Dis. 1-9. https://doi.org/ 10.1136/annrheumdis-2013-204599 (2014).

31. Vasheghani, F. et al. PPAR $\gamma$ deficiency results in severe, accelerated osteoarthritis associated with aberrant mTOR signalling in the articular cartilage. Ann. Rheum. Dis. 569-578 https://doi.org/10.1136/annrheumdis-2014-205743 (2015).

32. Settembre, C. et al. TFEB links autophagy to lysosomal biogenesis. Science $\mathbf{3 3 2}$ 1429-1433 (2011).

33. Bao, J. et al. Deacetylation of TFEB promotes fibrillar $A \beta$ degradation by upregulating lysosomal biogenesis in microglia. Protein Cell 7, 417-433 (2016).

34. Nakagawa, T. \& Guarente, L. Sirtuins at a glance. J. Cell Sci. 124, 833-838 (2011).

35. Liu-Bryan, R. \& Terkeltaub, R. Emerging regulators of the inflammatory process in osteoarthritis. Nat. Rev. Rheumatol. 11, 35-44 (2015).

36. Oppenheimer, $\mathrm{H}$. et al. 75-Kd Sirtuin 1 blocks tumor necrosis factor a-mediated apoptosis in human osteoarthritic chondrocytes. Arthritis Rheum. 64, 718-728 (2012).

37. Ben-Aderet, L. et al. Detecting cathepsin activity in human osteoarthritis via activity-based probes. Arthritis Res. Ther. https://doi.org/10.1186/s13075-0150586-5 (2015) 